\title{
Solución coordinada del problema de reconfiguración de alimentadores primarios y balance de fases
}

ELÉCTRICAL ENGINEERING

\section{Coordinated solution to the problem of primary feeder reconfiguration and phase balance}

\author{
Alejandro Garcés*, Ricardo A. Hincapié*, Ramón A. Gallego § \\ *Docentes Programa de Ingeniería Eléctrica-Universidad Tecnológica de Pereira, \\ Grupo de investigación en planeamiento de sistemas eléctricos \\ §Alejandro.garces@utp.edu.co,ricardohincapie@utp.edu.co,ragr@utp.edu.co
}

(Recibido: Agosto 26 de 2010 -Aceptado: Marzo 5 de 2012 -Versión Final: Junio 15 de 2012)

\begin{abstract}
Resumen
En este trabajo se presenta una metodología para reducir las pérdidas de energía en redes primarias de distribución, considerando un algoritmo combinado que involucra la reconfiguración óptima del sistema y el balance de fases. El modelo matemático es no lineal entero mixto, el cual tiene en cuenta los costos de inversión y operación de la red, sujeto a un conjunto de restricciones técnicas impuestas por el sistema. Para solucionar el problema de reconfiguración y balance de fases se emplean los algoritmos Simulated Annealing y Colonia de Hormigas, respectivamente. El algoritmo es comprobado usando el sistema IEEE de 37 nodos.
\end{abstract}

Palabras Claves: Balance de fases, reconfiguración, optimización combinatorial, sistemas de distribución.

\begin{abstract}
This paper presents a methodology to reduce energy losses in primary distribution networks, considering a combined algorithm which involves the optimal reconfiguration of the system and phase balance. The mathematical model is nonlinear mixed integer, which takes into account the investment and operating costs of the network, subject to a set of technical constraints imposed by the system. To solve the problem of reconfiguration and phase balance algorithms are used Simulated Annealing and Ant Colony, respectively. The algorithm is tested on IEEE 37 node feeder.
\end{abstract}

Keywords: Phase balancing, reconfiguration, combinatorial optimization, distribution systems. 


\section{Introducción}

De los sistemas que componen las redes eléctricas, son los sistemas de distribución los que presentan los índices más altos de pérdidas técnicas y notécnicas de energía. Debido a esto actualmente el esfuerzo de las empresas de distribución está centrado en la eliminación de las pérdidas notécnicas y en la reducción de las pérdidas técnicas. Por esta razón, las empresas distribuidoras de energía eléctrica requieren operar los sistemas con criterios técnicos de economía, confiabilidad y seguridad apropiados para garantizar la calidad del servicio a los clientes, cumpliendo con las normas técnicas exigidas y mínimo tiempo de interrupción del servicio, buscando siempre tener el menor índice posible de pérdidas en el sistema.

Debido a que en Colombia los sistemas de distribución en su mayoría no cuentan con sistemas SCADA (supervisory control and data acquisition), los cambios en su topología no son efectuados en tiempo real y por el contrario cada cambio debe permanecer por largos periodos de tiempo. Dado lo anterior, se requiere de una metodología que considere la permanencia de la configuración en un periodo de tiempo y modelado a través de una curva de duración de carga.

Con el fin de minimizar las pérdidas técnicas en estos sistemas se han empleado diversas metodologías como balance de fases, instalación de condensadores, reguladores de tensión, repotenciación de conductores y reconfiguración, entre otros.

De las técnicas mencionadas anteriormente, dos de las metodologías más eficientes y de bajo costo de implementación para la reducción de las pérdidas técnicas son la reconfiguración de alimentadores primarios y el balance de fases.

La reconfiguración de alimentadores primarios ha sido tratada en la literatura especializada de diversas maneras y en su solución se han empleado diversas técnicas como branch and bound (Merlin \& Back, 1975), branch exchange (Baran \& Wu, 1989), simulated annealing (Jeon et al., 2002), búsqueda tabú (Zhang et al., 2007), algoritmos genéticos (Carreno et al., 2008) y heurísticas constructivas (Shirmohammadi \& Hong, 1989; Goswani \& Basu, 1992; Cinvalar et al., 1988). Shirmohammadi \& Hong (1989) y Goswani \& Basu (1992) implementan un flujo de potencia óptimo en redes enmalladas, para luego determinar la línea que debe quedar inactiva hasta obtener una configuración radial con las mínimas pérdidas. Cinvalar et al. (1998) proponen una fórmula para determinar la variación de las pérdidas después de transferir carga de un ramal a otro, escogiendo la alternativa que produce la mayor reducción en las pérdidas, y sugiriendo algunos criterios heurísticos para reducir el espacio de búsqueda. Raju \& Bijwe (2008) proponen un método que emplea índices de sensibilidad y heurísticas en dos fases. La primera fase comienza con todos los interruptores cerrados (red enmallada) y determina la línea a salir según el mejor índice de disminución de pérdidas respecto a la impedancia, hasta conseguir una red radial. En la segunda fase se efectúa un cambio de las líneas que salieron en la primera fase con otra línea de la vecindad de la configuración, identificada en la primera fase.

El problema de balance de fases también ha sido tratado por diversos autores y resuelto usando diversas metodologías. Zhu et al. (1998) presentan un algoritmo para resolver el problema del balance de fases que utiliza una aproximación lineal del problema y asume modelos de cargas de corriente constante. Zhu et al. (1999) proponen un modelo no lineal entero mixto y lo resuelven aplicando simulated annealing para una condición de demanda pico. Galvis et al. (2005) plantean una metodología para solucionar este problema usando un algoritmo de búsqueda tabú. Garcés et al. (2005) proponen una metodología basada en colonia de hormigas para solucionar el problema, el cual considera en el modelo matemático la curva de duración de carga del sistema para un período de tiempo determinado.

Con el fin de disminuir las pérdidas técnicas en sistemas de distribución, se propone en este trabajo una metodología que considera en forma combinada la reconfiguración de alimentadores primarios y el balance de fases. Es propuesto un modelo matemático no lineal entero mixto que considera en la función objetivo la minimización 
de costos de inversión y de operación teniendo en cuenta la curva de duración de carga del sistema y donde el modelo asume la permanencia de la configuración en un periodo determinado y tramos nuevos de red son propuestos. Sin embargo el modelo es general en el sentido que puede ser resuelto para un instante de tiempo y una condición de demanda, o para un periodo de duración de la curva de carga. El sistema es modelado de forma trifásica lo cual proporciona un mejor estimado de la operación real de la red. Para ello fue necesario la implementación de un flujo de carga trifásico y el modelamiento de los principales componentes del sistema de forma desbalanceada. Para solucionar los problemas de reconfiguración y balance de fases se emplean como técnica de solución simulated annealing y colonia de hormigas, respectivamente. Para verificar la validez de la metodología propuesta se emplea el sistema IEEE de 37 nodos.

El artículo es organizado de la siguiente forma: inicialmente se presenta la metodología, la cual incluye la descripción del problema, el modelo matemático que describe los problemas de reconfiguración de alimentadores primarios y balance de fases, y las técnicas empleadas en la solución de dichos problemas. Posteriormente es presentada la aplicación a un sistema de prueba empleado en la literatura especializada. Finalmente se muestran las conclusiones más relevantes obtenidas en este trabajo.

\section{Metodología}

\subsection{Descripción del problema}

Tanto el problema de reconfiguración como de balance de fases corresponden a problemas de cambio de la topología del sistema, y debido a la cantidad de variables y alternativas que presentan en su solución, estos problemas son considerados como de gran complejidad combinatorial.

E1 problema de la reconfiguración de alimentadores primarios consiste en encontrar una configuración radial óptima para la operación del sistema, definiendo como objetivo las pérdidas, cargabilidad de las líneas, confiabilidad, etc., con determinadas líneas en operación y otras para respaldo de la operación (suplencias), a partir de la apertura o cierre de interruptores en las líneas. En otras palabras, la reconfiguración busca la mejor topología del sistema, según sus líneas, respecto a alguno de los objetivos planteados (o una combinación de varios). Por lo tanto, la reconfiguración de redes de distribución define cuales líneas del sistema deben estar activas y cuales inactivas para que el sistema funcione de forma óptima. Adicionalmente a la reducción de pérdidas técnicas, con la reconfiguración se obtiene un alivio en la cargabilidad de las líneas y el mejoramiento del perfil de tensión del sistema.

El problema del balance de fases consiste en hallar una configuración adecuada de conexión de las cargas para las tres fases del sistema, con el fin de garantizar que la corriente que circula por estas sea aproximadamente igual. Este equilibrio de las corrientes del sistema garantiza una reducción del nivel de pérdidas técnicas del sistema y un mejoramiento en el comportamiento de las protecciones debido a valores de corrientes no adecuadas que circulan por el neutro del sistema. Este problema puede ser tratado empleando la demanda máxima o la curva de carga del sistema.

\subsubsection{Modelo matemático}

El modelo matemático que describe el problema de la reconfiguración y balance de fases en forma conjunta es el siguiente: Ec. (1), (2), (3), (4), (5), (6), (7),

$$
\min \left(\sum_{\mathrm{k}} C_{\mathrm{k}} \times L_{\mathrm{k}}\right)+\left(C_{\mathrm{T}} \times \sum_{\mathrm{t}=1}^{\mathrm{T}} h_{\mathrm{t}} \times \sum_{\mathrm{i}=1}^{\mathrm{N}} R_{\mathrm{i}} \times \frac{P_{\mathrm{it}}^{2}+Q_{\mathrm{it}}^{2}}{V_{\mathrm{it}}^{2}}\right)+\left(C_{\mathrm{p}} \times P_{\mathrm{pp}}\right)
$$




$$
\begin{aligned}
\text { sujeto a: } & P_{\mathrm{it}}=\mathrm{f}_{\mathrm{P}}\left(V_{\mathrm{it},} \Theta_{\mathrm{it}}\right) \\
& Q_{\mathrm{it}}=\mathrm{f}_{\mathrm{Q}}\left(V_{\mathrm{it},} \Theta_{\mathrm{it}}\right) \\
& P_{\text {min }}<P_{\mathrm{it}}<P_{\max } \\
& V_{\min }<V_{\mathrm{it}}<V_{\max } \\
& H_{\mathrm{j}} \in\{1, \ldots, 6\} \\
& H_{\mathrm{j}} \leqslant 3 \quad \forall \mathrm{j}=\{\text { Carga motriz }\} \\
& \prod \lambda_{\mathrm{F}}=1
\end{aligned}
$$

En la función objetivo descrita en la Ec. (1), el primer término corresponde a los costos de inversión asociados a la construcción de nuevas suplencias. Estos tramos de línea son propuestos de acuerdo a criterios particulares de cada empresa y a las posibilidades geográficas y físicas del sistema. De esta forma la metodología no solo determina las líneas a operar sino también las posibles líneas a construir o interruptores a instalar. El segundo término representa los costos de operación del sistema, los cuales involucran las pérdidas de energía del sistema durante el periodo de planeamiento considerado. El último término considerada los costos de potencia pico en el sistema.

La Ec. (2) representa las ecuaciones de balance de potencia activa y reactiva inyectada, las cuales obedecen a un modelamiento trifásico. Los límites de operación del sistema son establecidos mediante las Ec. (3) y (4). Las Ec. (5) y (6) describen el conjunto de configuraciones de las fases del sistema. La ecuación (7) representa la restricción de radialidad del sistema resultante, donde $\lambda_{\mathrm{F}}$ es el número de caminos posibles entre cada uno de los nodos del sistema y el nodo fuente (slack).

El modelo matemático descrito por las Ec. (1) a (7) presenta una alta no-linealidad además de una alta complejidad matemática. Este modelo es general ya que permite realizar la reconfiguración bajo el enfoque convencional de las pérdidas de potencia para la demanda máxima, donde sólo es necesario introducir una curva de carga constante y hacer cero las constantes $C_{\mathrm{T}}$ y $C_{\mathrm{k}}$, o bajo el enfoque de la curva de duración de carga.
En el problema del balance de fases las variables de decisión corresponden a las configuraciones de $H$, tal como se muestra en la tabla 1 . La configuración $H$ implica los cambios a realizarse en cada carga, donde una disposición BCA indica que la carga conectada a la fase A pasa a la fase B, la de la fase $B$ pasa a la fase $C$, y la de la fase $C$ pasa a la fase A. Estos cambios se realizan teniendo en cuenta no modificar la secuencia de fase en las cargas motrices, como se expresa en la ecuación (6). Es importante aclarar que algunas cargas pueden permanecer inalteradas lo cual se manifiesta con un valor de $H$ igual a la unidad.

Tabla 1. Configuraciones de las cargas.

\begin{tabular}{cc}
\hline Configuración & $\mathrm{H}$ \\
\hline $\mathrm{ABC}$ & 1 \\
$\mathrm{BCA}$ & 2 \\
$\mathrm{CAB}$ & 3 \\
$\mathrm{CBA}$ & 4 \\
$\mathrm{ACB}$ & 5 \\
$\mathrm{BAC}$ & 6 \\
\hline
\end{tabular}

\subsubsection{Curva de carga}

Los diferentes usuarios conectados al sistema de distribución presentan una característica de consumo variable en el tiempo la cual es predecible, por lo menos a nivel de alimentador primario (a partir de consumos históricos), y relativamente estable debido a la superposición de las cargas. Esta característica es representada mediante la curva de carga la cual puede ser diaria, mensual o anual. El uso de la curva de carga permite que un problema puramente operativo como la reconfiguración o el balance de fases sea llevado a un horizonte de planeamiento. Sin embargo, las cargas del sistema son de distinta naturaleza (residencial, comercial, industrial, etc), razón por la cual es aconsejable tener información detallada para cada tipo de usuario.

En los sistemas de distribución reales la falta de un adecuado seguimiento de la demanda desagregando el tipo de carga, hace necesario utilizar una única curva para cada alimentador o subestación. En este caso en particular puede ser utilizada la curva de duración de carga discretizada en $\mathrm{T}$ intervalos de tiempo. El valor más usual de $\mathrm{T}$ es de tres a cinco intervalos. La metodología propuesta permite tener en cuenta 
ambas posibilidades (con curva de carga individual o curva de duración de carga global).

\subsubsection{Modelamiento de los elementos del sistema}

Dependiendo de las condiciones de operación y de diseño, los sistemas de distribución son analizados a través de representaciones que pueden ser trifásicas o monofásicas equivalentes. El primero es propio de sistemas con operación desequilibrada mientras que el segundo es usado para sistemas que operan en forma equilibrada o aquellos en donde el desequilibrio de las fases no afecta significativamente los resultados. En el caso de la representación trifásica, los resultados son más exactos aunque se requiere gran cantidad de información acerca de las cargas y un modelo detallado que incluya los efectos del neutro. El segundo caso corresponde a una aproximación que permite encontrar resultados aceptables en sistemas con índice de desbalance reducido.

Como los sistemas de distribución generalmente presentan un alto índice de desequilibrio, se pretende en este trabajo mejorar esto con el fin de disminuir las pérdidas y reducir la corriente por el neutro. Debido a lo anterior, los elementos del sistema fueron modelados en forma trifásica, de acuerdo a los parámetros descritos por Kersting (2007).

\subsection{Técnicas de solución}

\subsubsection{Flujo de carga}

Debido al modelamiento trifásico de los elementos del sistema, es empleado un flujo de carga radial trifásico de barrido iterativo. Este método se basa en las leyes de Kirchhoff y evita el uso de Jacobianos, obtieniendo soluciones de igual calidad y en un tiempo inferior al método de Newton-Raphson.

\subsubsection{Reconfiguración de alimentadores primarios}

El problema de reconfiguración de alimentadores primarios fue solucionado utilizando una metodología basada en el método simulated annealing. El algoritmo inicia con un parámetro $T$ (temperatura) que mide la probabilidad de aceptación de soluciones de mala calidad. En general las soluciones de buena calidad son aceptadas y permiten el cambio de la incumbente. Las soluciones que no producen una mejora en la función objetivo son aceptadas con una probabilidad dada por la siguiente ecuación:

$$
P_{\text {(aceptar) }}=\mathrm{e}^{\left(f_{\mathrm{i}}-f_{\mathrm{j}}\right) / T}
$$

En la expresión anterior $f_{\mathrm{i}}$ representa la función objetivo para el estado de operación i mientras $f_{\mathrm{j}}$ representa la función objetivo para el estado de operación siguiente j. La transición entre el estado i y el estado j se realiza mediante un criterio de vecindad previamente establecido. La función objetivo incluye el costo de las pérdidas de potencia y de energía y el costo de nuevas suplencias, la cual es evaluada mediante el flujo de carga radial.

El valor de $T$ es disminuido al inicio de cada cadena, de esta forma la probabilidad de aceptar alternativas de peor calidad disminuye. A este proceso se le conoce como programa de enfriamiento y se puede efectuar por medio de una taza de enfriamiento $\eta$ (generalmente entre 0.80.99), descrita a continuación:

$$
T_{\mathrm{k}+1}=\eta T_{\mathrm{k}}
$$

La longitud de la cadena se estima inicialmente de acuerdo al tamaño del problema. Una de esas formas es asumirla como $\mathrm{k}$ veces el número de líneas del sistema. Existe un valor determinado de vecinos a ser evaluados para cada valor de $T$, el cual es llamado número de tentativas $N_{\mathrm{k}}$.

En cada ciclo la longitud de la cadena debe aumentar su tamaño, donde este parámetro $(\rho)$ es mayor a 1 y su valor debe ser calibrado. Por lo tanto:

$$
N_{\mathrm{k}+1}=\rho N_{\mathrm{k}}
$$

El criterio de vecindad se refiere a la regla heurística que determina la configuración siguiente en el proceso iterativo. En este trabajo se empleó el método branch Exchange, el cual elige una suplencia a ser ubicada en el sistema de 
acuerdo a un criterio aleatorio o un índice de sensibilidad. La elección entre estos dos criterios mencionados, es determinado de forma estocástica de acuerdo a una probabilidad $P_{\text {in }}$ definida a priori. Al conectar una suplencia en el sistema radial se forma una malla que debe ser abierta para conservar la radialidad, para lo cual se elige una rama de esta malla que pasa a ser la nueva suplencia. La elección de esta rama es realizada nuevamente de acuerdo a los criterios mencionados.

Este tipo de estrategia permite utilizar la potencialidad de la metodología combinatorial, con la capacidad de los índices de sensibilidad de encontrar rutas promisorias. El índice de sensibilidad elegido para la entrada de una suplencia está descrito por:

$$
\sigma=\frac{V_{\mathrm{ab}}^{2}}{R_{\mathrm{ab}}}
$$

Donde $\sigma, V_{\mathrm{ab}}$ y $R_{\mathrm{ab}}$ son el índice de reducción de pérdidas al cerrar el enlace ab, el voltaje en terminales del enlace $a b$ antes de realizar el cambio y la resistencia del tramo de línea $a b$, respectivamente. El índice para definir la rama de la malla a salir del sistema radial es la mínima magnitud de la corriente por las ramas de la malla.

La temperatura inicial es definida de acuerdo a la siguiente ecuación:

$$
T_{0}=\frac{D_{\mathrm{vi}}}{\ln \left(P_{\mathrm{Ai}}\right)} \times f_{0}
$$

Donde $D_{\text {vi, }}, P_{\text {Ai, }}, f_{0}$ y $T_{0}$ son la desviación inicial permitida de la función objetivo, la probabilidad de aceptación inicial, la función objetivo del sistema base y la temperatura inicial, respectivamente.

\subsubsection{Balance de fases}

El algoritmo de balance de fases se desarrolla basado en la metodología de colonia de hormigas. Esta metodología modela el rastro químico dejado por las hormigas en su proceso de encontrar alimento. Dicho rastro, denominado feromona, sirve como indicativo a las siguientes hormigas para realizar su búsqueda. En el caso matemático, el rastro químico es modelado por una matriz que determina la probabilidad de elegir determinado camino por parte de las diferentes hormigas. A medida que la función objetivo mejora, los rastros de feromona se hacen más fuertes para indicar trayectorias que conllevan a soluciones de alta calidad. Existe igualmente un elemento denominado evaporación que disminuye este rastro con el fin de dar diversidad al método y evitar los óptimos locales.

La matriz de feromonas $(F)$ es de tamaño $6 \times N_{\text {C }}$, en donde $N_{\mathrm{C}}$ es el número de cargas del sistema. Cada elemento $F_{\text {hj }}$ representa el nivel promedio de mejoría de la función objetivo cuando se modifica la carga j implementando la configuración h. Este valor es actualizado en cada iteración de acuerdo a un índice de desbalance $\delta$ definido por la siguiente ecuación:

$$
\delta=\frac{\operatorname{Max}\left\{\left|I_{\mathrm{a}}-I_{\text {prom }}\right|,\left|I_{\mathrm{b}}-I_{\text {prom }}\right|,\left|I_{\mathrm{c}}-I_{\text {prom }}\right|\right\}}{I_{\text {prom }}} \times 100 \%
$$

En la expresión anterior $I_{\text {prom }}, I_{\mathrm{a}}, I_{\mathrm{b}} \mathrm{e} I_{\mathrm{c}}$ representan la corriente promedio de las tres fases, y las corrientes por las fases a, b y c, respectivamente. El valor de este índice es cero cuando existe un balance total y sirve tanto para guiar el proceso de optimización como para indicar la calidad de la solución.

Cada hormiga corresponde a una configuración $H$ de las diferentes cargas. En cada iteración, se realizan cambios en las cargas de acuerdo a dos criterios: explotación (elige de forma aleatoria una de las seis posibles configuraciones de cada carga teniendo en cuenta la probabilidad dada por la matriz de feromonas) y exploración (elige la configuración que presente mayor valor en la matriz de feromonas). La función de cada hormiga consiste en recorrer cada una de las cargas y establecer su conexión de acuerdo a uno de estos dos criterios. El criterio en cada carga se determina con una regla probabilística dada por un parámetro definido previamente. Este parámetro se fija en el intervalo [0-1] de manera que si es igual a 0.5 existe la misma probabilidad de realizar explotación o exploración. Por otra parte, un valor 
cercano a cero implica una escasa probabilidad de realizar exploración lo que hace el procedimiento altamente determinístico, el cual puede conducir eventualmente a una convergencia temprana en un óptimo local.

En el proceso de exploración, la configuración $H_{\mathrm{j}}$ se elige utilizado el método de la ruleta. La probabilidad asociada a cada configuración está dada por la Ec.(14):

$$
P_{\mathrm{jh}}=\frac{F(\mathrm{j}, \mathrm{h})}{6 \times \sum_{\mathrm{h}=1}^{6} F(\mathrm{j}, \mathrm{h})}
$$

En este proceso se genera un número aleatorio entre 0 y 1 el cual se compara con la probabilidad acumulada asociada con cada una de las 6 configuraciones. La configuración con mayor $F_{\text {jh }}$ tiene mayor probabilidad de ser seleccionada. En cada recorrido puede ocurrir que la solución elegida sea no factible al violar la restricción de cambio de secuencia. En este caso la nueva configuración debe ser factibilizada restando 3 al valor de $H$ correspondiente a la carga motriz.

Finalizado este proceso, se calcula la función objetivo mediante la ejecución del flujo de carga trifásico. Si existe una reducción en las pérdidas que mejore la calidad de la mejor solución encontrada hasta el momento (incumbente), se actualiza tanto la incumbente como la matriz de feromonas. En este caso el aumento en la matriz de feromonas $\left(D_{\mathrm{jh}}\right)$ es constante y definido a priori, donde el nuevo valor en la matriz se expresa como:

$$
F_{\text {jh }}=F_{\text {jh }} \times E_{\mathrm{v}}+D_{\text {jh }}
$$

Donde $E_{\mathrm{v}}$ es el coeficiente de evaporación y representa el grado de "olvido", el cual proporciona al método mayor diversidad.

Si la nueva configuración, producto del recorrido de la hormiga, presenta una función objetivo de peor calidad que la incumbente, se actualizan solo los valores en los nodos en los cuales hay una reducción en el índice de desbalance. El procedimiento global continúa durante un número de iteraciones predeterminado mientras que el número de hormigas de la colonia determina el número de recorridos globales por cada iteración.
Si cumplido un número de iteraciones no se produce ningún cambio en la incumbente, se aplica un proceso de diversificación, consistente en reemplazar por un número aleatorio los rastros de feromona en aquellos nodos donde el índice de desbalance sea mayor al 5\%, y se remplaza por uno los demás nodos.

\subsubsection{Método combinado}

Esta metodología, denominada esquema vertical, da solución de forma consecutiva a los problemas de reconfiguración y balance de fases. La solución óptima a uno de los problemas planteados se ve afectada por los cambios realizados por la otra metodología, por tal razón, es necesario ejecutar el algoritmo de reconfiguración después de realizado el de balance de fases, y viceversa. Es importante resaltar que el orden y número de etapas a considerar puede afectar la optimalidad de la solución encontrada. Los diagramas de bloques de la metodología planteada se ilustran en la figura 1.

\section{Resultados y discusión}

Estas metodologías fueron implementadas en el lenguaje de programación Delphi 7.0. Se utilizó el sistema IEEE de 37 nodos para verificar la validez de la metodología propuesta (figura 2). Este sistema presenta cargas desbalanceadas, líneas subterráneas y transformadores de distribución. Las líneas activas en el sistema se ilustran con líneas continuas y las suplencias con líneas discontinuas, las cuales corresponden a una configuración 722 de $0.1 \mathrm{~km}$ y un costo de 1,915 [\$/m]. Este sistema (sistema base) presenta unas pérdidas de potencia trifásica de $33.24 \mathrm{~kW}$.

En las tablas 2 y 3 se presentan los parámetros utilizados para el problema de reconfiguración y de balance de fases, respectivamente. En la tabla 2, los tres primeros datos están relacionados con el cálculo de la temperatura inicial. Los siguientes cuatro parámetros son el producto de la calibración de los mismos, es decir, se determinaron después de realizar varios ensayos. En la tabla 3 los primeros cinco parámetros son el resultado de la parametrización del algoritmo, los cuales fueron definidos después de efectuar varias pruebas. 


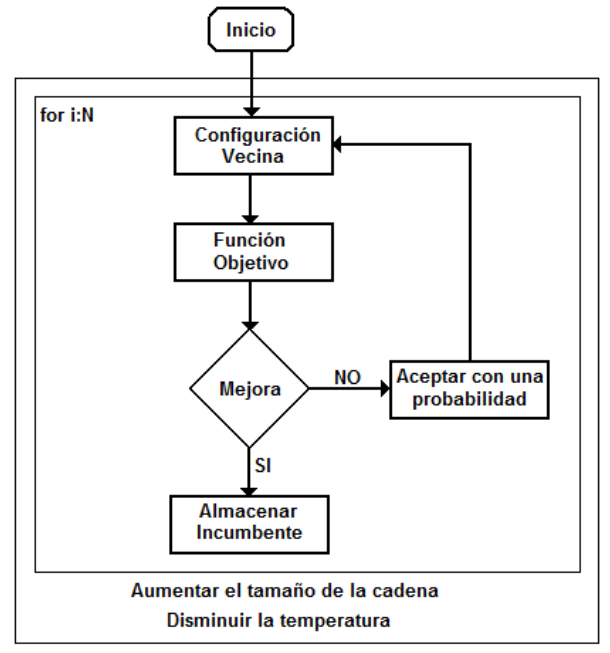

a. Simulated Annealing.

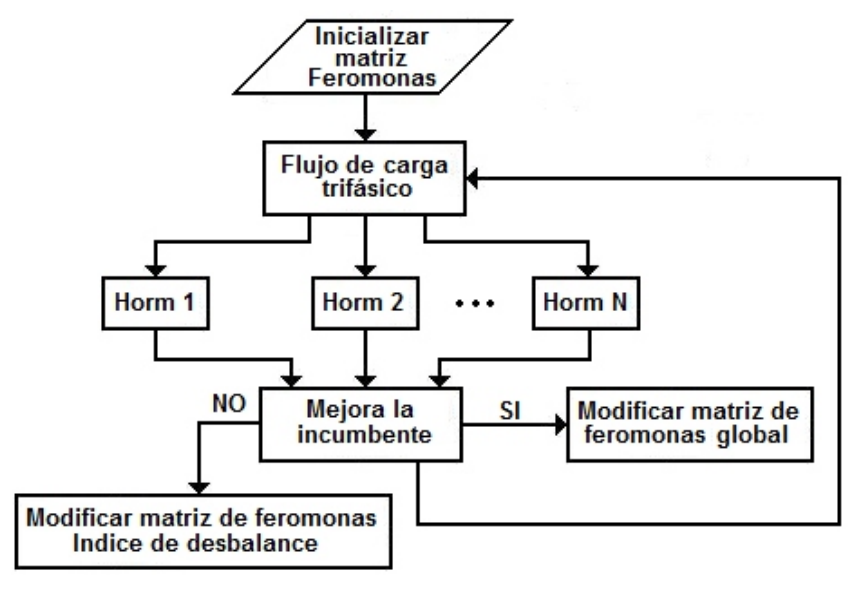

b. Colonia de Hormigas.

Figura 1. Diagrama de bloques de los algoritmos de solución.

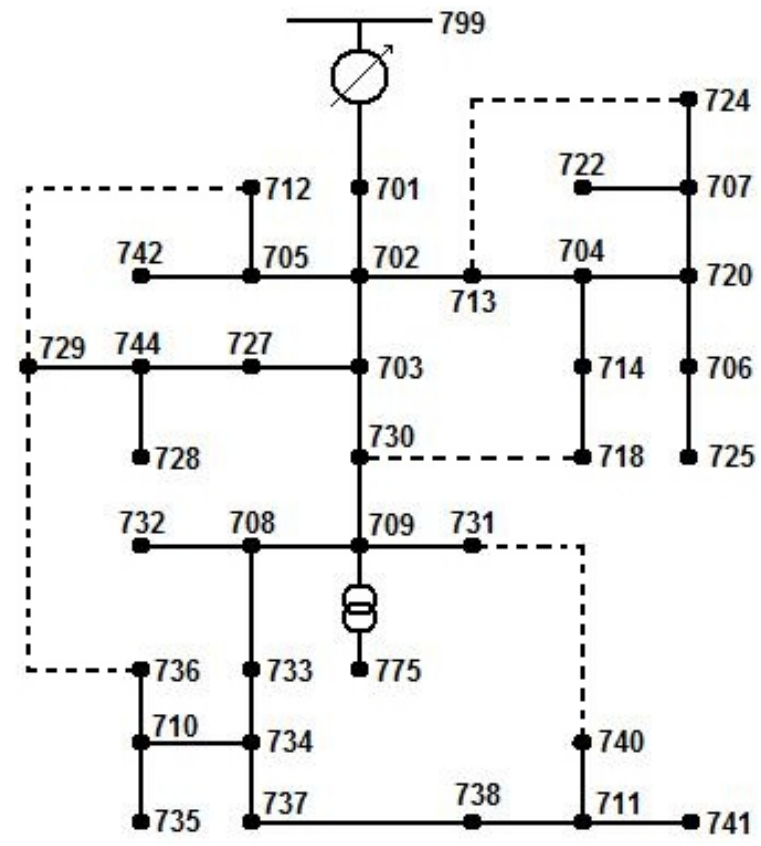

Figura 2. Sistema IEEE de 37 nodos.

La curva de duración de carga aplicada a todas las cargas del sistema es mostrada en la tabla 4. Esta curva está discretizada en tres niveles de demanda (máxima, media y mínima), debido a que presenta buenos resultados y se ajustar bien al comportamiento de la carga.
Tabla 2. Parámetros empleados en la reconfiguración.

\begin{tabular}{cc}
\hline Parámetro & Valor \\
\hline Probabilidad de entrada determinística $\left(P_{\text {in }}\right)$ & $60 \%$ \\
Desviación inicial $\left(D_{\mathrm{vi}}\right)$ & $20 \%$ \\
Probabilidad de aceptación inicial $\left(P_{\mathrm{Ai}}\right)$ & $30 \%$ \\
Tamaño de la cadena $\left(\mathrm{N}_{\mathrm{o}}\right)$ & 500 \\
Tasa de enfriamiento $(\eta)$ & 0.8 \\
Aumento de la cadena $(\rho)$ & 1.1 \\
Número de estados & 10 \\
\hline
\end{tabular}

Tabla 3. Parámetros empleados en el balance de fases.

\begin{tabular}{cc}
\hline Parámetro & Valor \\
\hline Número de hormigas & 10 \\
Número de iteraciones & 30 \\
Índice de exploración & 0.8 \\
Nivel de evaporación & 0.7 \\
Número máximo de cambios & 25 \\
Número de cargas motrices & 0 \\
\hline
\end{tabular}

Tabla 4. Curva de duración de carga.

\begin{tabular}{cc}
\hline Duración [h] & Valor [\%] \\
\hline 1,000 & 100 \\
3,000 & 60 \\
4,760 & 30 \\
\hline
\end{tabular}


La configuración final del sistema después de aplicar la metodología propuesta se observa en la figura 3, donde las líneas que quedaron como suplencias en la configuración final son: 704-713, 703-730, 731-740, 703-727, 710-736. En la tabla 5 se presentan los nodos a los cuales se les efectuó un cambio en las fases, mostrando la configuración final de estas.

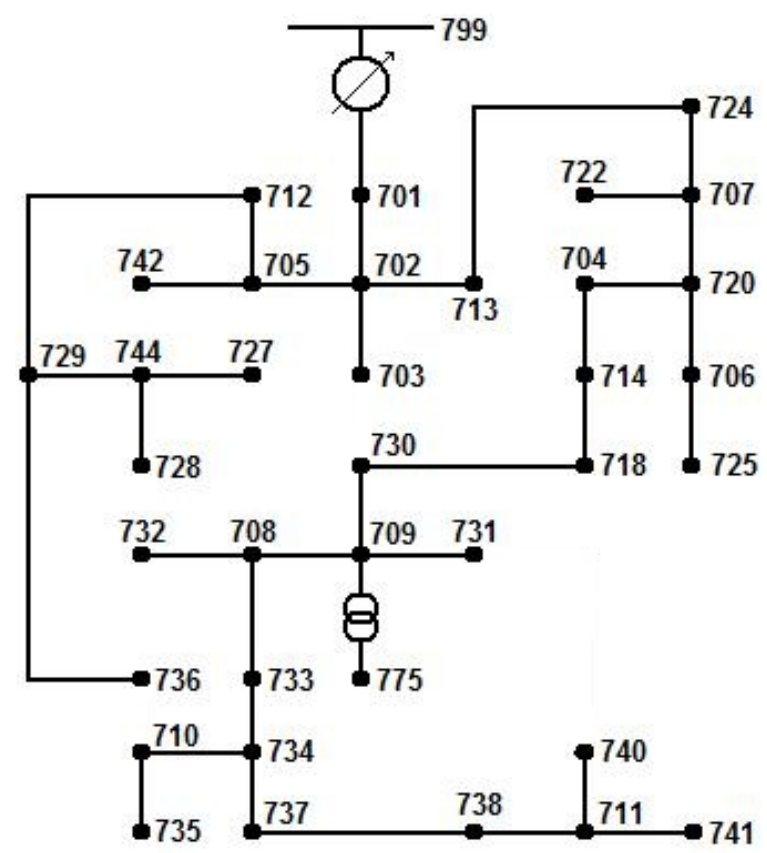

Figura 3. Configuración final del sistema.

Tabla 5. Cambios del balance de fases.

\begin{tabular}{cc}
\hline Cambio & Nodos \\
\hline ACB & $713-730-741$ \\
CAB & $714-724-727-728-732-733-738-740-744$ \\
CBA & $718-734-737$ \\
BCA & $720-736$ \\
BAC & $722-731-742$ \\
\hline
\end{tabular}

Los resultados obtenidos se ilustran en la tabla 6, en la cual se comparan los valores entregados por la metodología después de realizar diversos casos de prueba con respecto al sistema base (sistema inicial). En el caso 1 solamente se considera el balance de fases, en el siguiente caso solamente se aplica reconfiguración, y en el último caso se aplican las dos estrategias en forma simultánea.

Se puede observar que ambas estrategias empleadas en forma separada son eficientes. Sin embargo, considerarlas en forma conjunta reduce aún más las pérdidas técnicas del sistema, que aplicarlas en forma independiente. Con la aplicación de la metodología propuesta se logra una reducción de pérdidas del sistema del $28.27 \%$ con respecto al sistema inicial, lo cual impacta positivamente en la operación del sistema y las finanzas del operador de red.

\section{Conclusiones}

En este trabajo se propuso e implementó una metodología para reducir las pérdidas técnicas en sistemas de distribución, la cual usa en forma coordinada la reconfiguración de alimentadores primarios y el balance de fases, y para su solución se emplean las técnicas de los algoritmos simulated annealing y colonia de hormigas, obteniendo resultados de excelente calidad.

Al resolver el problema de reconfiguración y de balance en forma independiente se obtienen mejoras significativas en el nivel de pérdidas respecto al caso base. Sin embargo cuando el problema es resuelto en forma coordinada usando reconfiguración y balance de fases, se obtienen mejoras adicionales respecto al nivel de pérdidas base, con lo cual se concluye que los problemas planteados al ser independientes tienen que ser resueltos en forma coordinada.

Tabla 6. Resultados obtenidos.

\begin{tabular}{cccc}
\hline Descripción & $\begin{array}{c}\text { Pérdidas de } \\
\text { potencia en kW }\end{array}$ & $\begin{array}{c}\text { Disminución de las } \\
\text { pérdidas en } \%\end{array}$ & Observaciones \\
\hline Sistema base & 33.24 & ---- & Sistema inicial \\
Caso 1 & 30.24 & 9.15 & Usando solamente balance de fases \\
Caso 2 & 25.8 & 22.3 & $\begin{array}{c}\text { Empleando solamente reconfiguración } \\
\text { Aplicando reconfiguración y balance de } \\
\text { faso 3 }\end{array}$ \\
Caso en forma conjunta
\end{tabular}


Las técnicas de optimización simulated annealing y colonia de hormigas utilizadas en la solución de los problemas de reconfiguración y balance de fases, usan tipos de codificación que son de fácil implementación y presentan excelentes resultados para este tipo de problemas.

En sistemas de distribución no automatizados como es la gran mayoría de los sistemas en Colombia, el análisis se lleva a cabo en un periodo de tiempo, usando una curva de duración de carga correspondiente al periodo para el cual se fija la topología establecida, tanto para el problema de reconfiguración como para el de balance de fases. En este problema además de minimizar la inversión en tramos de red, se minimiza el nivel de pérdidas de energía y de potencia en el periodo establecido.

Como alternativa de solución al modelo matemático planteado en las Ec. (1) a (7) se tendría un esquema denominado horizontal. En este, los problemas de reconfiguración y balance de fases se resuelven al tiempo y no tendría que esperar a resolver un problema para iniciar el siguiente. Las acciones de reconfiguración y balance son propuestas de manera simultánea.

\section{Nomenclatura}

$C_{\mathrm{k}}$ : costo de construcción de la suplencia k.

$C_{\mathrm{p}}$ : costo de la demanda máxima.

$C_{\mathrm{T}}$ : costo de la energía comprada.

$h_{\mathrm{t}}$ : duración en horas del periodo de tiempo t.

$H_{\mathrm{j}}$ : índice de la configuración de cada carga.

i : índice de las líneas del sistema radial.

$\mathrm{j}$ : índice de las cargas del sistema.

$\mathrm{k}$ : índice de las suplencias a construir.

$L_{\mathrm{k}}$ : variable de decisión que indica si se construye o no la suplencia $\mathrm{k}$.

$P_{\mathrm{it}}$ : flujo de potencia activa por la línea i en el periodot.

$P_{\min }, P_{\max }:$ límites de potencia mínima y máxima permitida, respectivamente.
$P_{\mathrm{pp}}:$ pérdidas de potencia en el nivel de demanda pico.

$Q_{\text {it }}$ : flujo de potencia reactiva por la línea i en el periodot.

$R_{\mathrm{i}}$ : resistencia del tramo i de la red radial.

t: índice de los periodos de tiempo considerados.

T: periodo de tiempo considerado.

$V_{\text {it }}$ : magnitud del voltaje en el nodo correspondiente al envío en la rama $i$ en el periodo de tiempot.

$\Theta_{\text {it }}$ : ángulo del voltaje en el nodo correspondiente al envío en la rama $i$ en el periodo de tiempot.

$V_{\min }, V_{\max }$ : límites de voltaje mínimo y máximo permitido, respectivamente.

\section{Referencias bibliográficas}

Baran, M., \& Wu, F. (1989). Network reconfiguration in distribution system for loss reduction and load balancing. IEEE Transactions on Power Delivery 4 (2), 1401-1407.

Carreno, E., Romero, R., \& Padilha, A. (2008). An Efficient Codification to Solve Distribution Network Reconfiguration for Loss Reduction Problem. IEEE Transactions on Power Systems 23 (4), 1542-1551.

Cinvalar, S., Grainger, J., Yin, H., \& Lee, S. (1988). Distribution feeder reconfiguration for loss reduction. IEEE Transactions on Power Delivery 3 (3), 1217-1223.

Galvis, M., Granada, M., \& Gallego, R. (2005). Reducción del desbalance en sistemas de distribución aplicando búsqueda tabú. Simposio Internacional sobre la Calidad de la Energía Eléctrica (SICEL), Bogotá, Colombia.

Garcés, A., Granada, M., \& Gallego, R. (2005). Balance de fases usando colonia de hormigas. Revista Ingeniería y Competitividad 7, 43-52.

Goswami, S., \& Basu, S. (1992). A new for the reconfiguration of distribution feeders for loss minimization. IEEE Transactions on Power Delivery 7 (3), 1484-1491. 
Jeon, Y., Kim, J.C., Kim, J.C., Shin, J., \& Lee, K. (2002). An efficient simulated annealing algorithm for network reconfiguration in largescale distribution systems. IEEE Transactions on Power Delivery 17 (4), 1070-1078.

Kersting, W.H. (2007). Distribution system modeling and analysis. CRC Press, 2 nd. ed.

Merlin, A., \& Back, G. (1975). Search for minimum-loss operational spanning tree configuration for an urban power distribution system. In Proceedings of the 5th Power System Conference (PSCC), Cambridge, United Kingdom, p. 1-18.

Raju, G., \& Bijwe, P. (2008). An Efficient Algorithm for Minimum Loss Reconfiguration of Distribution System Based on Sensitivity and Heuristics. IEEE Transactions on Power Systems 23 (3), 1280-1287.

Shirmohammadi,D., \& Hong, H. (1989). Reconfiguration of electric distribution networks for resistive line losses reduction. IEEE Transactions on Power Delivery 4 (2), 1492-1498.

Zhang, D., Fu, Z., \& Zhang, L. (2007). An improved TS algorithm for loss minimum reconfiguration in large-scale distribution systems. Electric Power system Research 77 (5), 685-694.

Zhu, J., Griff, B., \& Chow, M. (1998). Phase balancing using mixed-integer programming. IEEE Transactions on Power Systems 13 (4), 1487-1492.

Zhu, J., Griff, B., \& Chow, M. (1999). Phase balancing using simulated annealing. IEEE Transactions on Power Systems 14 (4), 15081513. 